\section{Cutaneous Leishmaniasis caused by L. Infantum}

Sir,

Cutaneous leishmaniasis (CL) is endemic in different regions of Pakistan and the estimated disease incidence is 21,000 to 35,000 cases per year. ${ }^{1}$ Both anthroponotic $(A C L)$ and zoonotic (ZCL) forms of $C L$ are prevalent. ACL reportedly caused by Leishmania (L.) tropica, occurs sporadically, characterized by dry-type lesions and is reported usually from the urban areas while ZCL is attributed to $L$. major, presenting as wet lesions and is commonly reported from rural and semi-urban areas of Punjab, Balochistan, and Sindh.2,3 L. infantum along with $L$. donovani are the two parasites known to cause visceral leishmaniasis. In addition to visceral disease L. infantum has also been incriminated occasionally as the causative species of cutaneous disease in countries of Mediterranean basin, surrounding Europe and Asian countries. ${ }^{4}$ But until recently, it was never reported from Pakistan.

Case 1: A 26-year soldier presented with 2-month history of gradually enlarging erythematous, painless and nonitchy nodular plaque on ventral aspect of his right wrist (Figure 1). He was a resident of Mirpurkhas (Sindh), but was deployed in Miran Shah (North Waziristan) for the last one and half years. Skin slit smear and parasite culture for Leishmania parasites were negative, but histopathology revealed LD bodies. It was identified as $L$. infantum using diagnostic PCR targeting KDNA (kinetoplast DNA) and ITS2 (Internal transcribed spacer 2 region) on clinical samples collected on filter papers. Patient was treated with weekly intralesional injections of meglumine antimonite and the lesion healed in three months.
Case 2: A 30-year soldier reported with 2-month history of gradually enlarging erythematous, crusted, painless and non-itchy plaque with occasional oozing on left external ear (Figure 2). He belonged to district Jhelum of Punjab, but was deployed in Miran Shah (North Waziristan) for the last 14 months. Skin slit smear, histopathology and culture for Leishmania parasites came out to be negative, but was identified as L. infantum through KDNA and ITS2 PCR. Patient was treated with weekly intralesional injections of meglumine antimonite satisfactorily.

Case 3: A60-year female, resident of Bagh (Azad Kashmir) presemted with asymptomatic erythematous dry infiltrated plaque on left cheek for the last 8 months (Figure 3). Considering the chronicity and morphology of the lesion, it was clinically suspected as a case of Lupoid CL.She was negative for Leishmaniasis using skin slit smear, histopathology and culture for Leishmania parasites, but PCR (KDNA and ITS2) proved $L$. infantum as the causative species. Patient was again treated with weekly intralesional injections of meglumine antimonite with favorable response.

In Pakistan, a substantial work has been done on clinical and epidemiological aspects of the disease, but many fail to conduct species level diagnosis.2-5 This has resulted in failure to make appropriate scientific comparisons, and inability to correlate clinical polymorphism with species diversity. Recently, Khan et al. did a species level study using species specific PCR screening and were able to identify $L$. infantum as causative species of $\mathrm{CL}$ in three cases. Two of these reported from the region not known for VL, but third case was a 60-year female from AJK (Azad Jammu Kashmir), which is a known focus of VL by $L$. infantum. 6

These three reported cases urge future studies for species typing of $\mathrm{CL}$ patients in KP and other provinces. Search for potential hosts and vectors for $L$. infantum in the region is also warranted in future research projects.

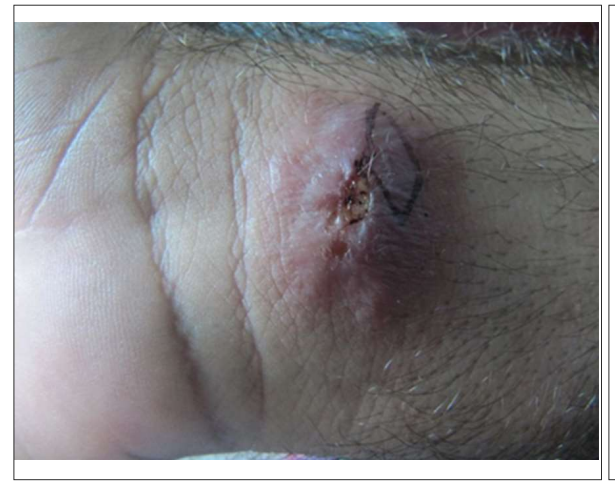

Figure 1: An erythematous nodular plaque with central ulceration on wrist region.

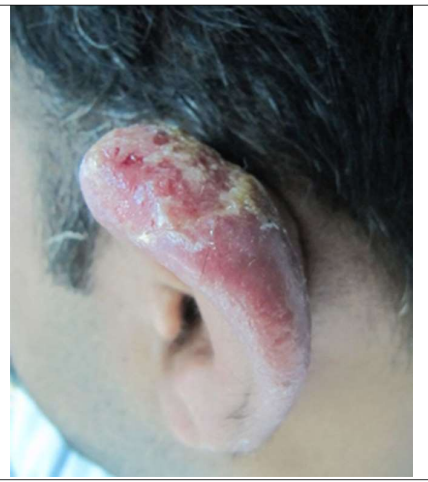

Figure 2: An erythematous plaque with partial ulceration and crusting on supero-lateral portion of external ear.

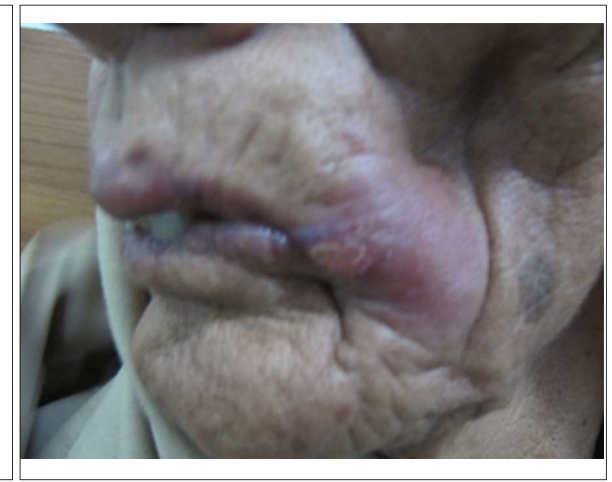

Figure 3: A solitary indurated plaque on left side of face adjacent to left oral commissure. 


\section{REFERENCES}

1. Alvar J, Vélez ID, Bern C, Herrero M, Desjeux P, Cano J, et al. Leishmaniasis worldwide and global estimates of its incidence. PLoS One 2012; 7:e35671.

2. Bari AU. Epidemiology of cutaneous leishmaniasis. J Pak Assoc Dermatol 2006; 16:156-62.

3. Afghan AK, Kassi M, Kasi PM, Ayub A, Kakar N, Marri SM. Clinical manifestations and distribution of cutaneous leishmaniasis in Pakistan. J Trop Med 2011; 2011:359145.

4. Svobodová M, Alten B, Zídková L, Dvorák V, Hlavacková J, Mysková $\mathrm{J}$, et al. Cutaneous leishmaniasis caused by Leishmania infantum transmitted by phlebotomus tobbi. Int $J$ Parasitol 2009; 39:251-6.

5. Khan NH, Bari AU, Hashim R, Khan I, Muneer A, Shah A, et al. Cutaneous leishmaniasis in Khyber Pakhtunkhwa province of
Pakistan; Clinical diversity and species-level diagnosis. Am J Trop Med Hyg 2016; 95:1106-14.

6. Rab MA, lqbal J, Azmi FH, Munir MA, Saleem M. Visceral Leishmaniasis: A seroepidemiological study of 289 children from endemic foci in Azad Jammu and Kashmir by indirect fluorescent antibody technique. J Pak Med Assoc 1989; 39: 225-8.

Arfan ul Baril and Nazma Habib Khan'2

1 Department of Dermatology, CMH, Malir Cantt, Karachi, Pakistan

2 Departmnet of Zoology, University of Peshawar, KPK, Pakistan Correspondence: Dr. Arfan ul Bari, Department of Dermatology, CMH, Malir Cantt, Karachi, Pakistan

E-mail: albariul@gmail.com

Received: May 16, 2016; Accepted: October 18, 2018 